\title{
A Mixed-Method Investigation into Therapeutic Yoga as an Adjunctive Treatment for People Recovering from Substance Use Disorders
}

\author{
Christopher Fitzgerald $^{1}$ (D) Ruth Barley $^{1} \cdot$ Joanna Hunt $^{1} \cdot$ Sini-Petriina Klasto $^{1}$ • \\ Rosie West ${ }^{1}$
}

Published online: 30 January 2020

(C) The Author(s) 2020

\begin{abstract}
Mind Body Connect (MBC) is a charity which uses therapeutic yoga as a vehicle of change for marginalized populations. Alongside MBC, Sheffield Hallam University's SHU Strength researchers carried out this study aiming to: (1) Gauge the impact of therapeutic yoga classes upon the mood state of people with a Substance Use Disorder (SUD) and (2) Explore the perceived benefits of therapeutic yoga class participation. An adapted shortened Profile of Mood States (POMS) was completed before and after each yoga class. A comparison of means with paired sample T-Test and Cohen's D was then carried out. Participants who attended 6+ classes were interviewed. Findings were then converged. Before and after measurements of anger, sadness, tiredness, worry, confusion, energy and relaxation were taken, Classes were held at SHU for service users from a Phoenix Future's (PF) rehabilitation centre. A single yoga class significantly relaxed participants and reduced negative mood states. Interview data covered a range of perceived benefits including the use of yogic down-regulation techniques as daily coping strategies. The MBC yoga programme appears beneficial as an adjunctive therapy for PF residents. Future SHU Strength research shall focus on the mid-long-term exercise habits of the recovery community and the impact of the $\mathrm{MBC}$ yoga programme upon the early recovery period of detoxification.
\end{abstract}

Keywords Recovery $\cdot$ Recovery capital $\cdot$ Addiction $\cdot$ Substance use disorder $\cdot$ Yoga $\cdot$ Exercise $\cdot$ POMS $\cdot$ Mood state $\cdot$ Physical exercise $\cdot$ Physical activity

Christopher Fitzgerald dscf@exchange.shu.ac.uk

1 Department of Psychology, Sociology and Politics, Room 2.06, Heart of the Campus, Collegiate Crescent, Sheffield Hallam University, Sheffield S10 2BQ, UK 


\section{Setting}

Founded in 1969, Phoenix Futures (PF) is a residential drug and alcohol rehabilitation centre, the first in the UK to operate under the Therapeutic Community model. The PF conceptualisation of recovery is based upon abstinence from all drugs but encompasses broader biopsychosocial development. Interpersonal skills and introspection are focused on during a 6 to 9-month hierarchical "Work as Therapy" and "Community as Method" programme (Phoenix Futures 2011).

Mind Body Connect (MBC) is a charity which runs a therapeutic yoga programme for PF's Sheffield-based centre. The MBC remit is to empower marginalized populations through the medium of exercise. MBC also ascribes to the assumption that recovery requires more than being substance-free and aims to increase service user capital stocks. Originally conceptualized by Cloud and Granfield (2008), this "recovery capital" is defined as the sum of economic, cultural, social and human resources an individual possesses, with which they can initiate and/ or sustain recovery. The recovery capital conceptual framework has proven useful for explaining the processes of identity reconstruction underpinning recovery (Best and Laudet 2010). This is achieved through the application of the classical Bourdieusian conceptualisation of capital, with the addition of a human capital subcategory to account for 'self-identity, confidence in expressing one's own opinions, and emotional intelligence' (Gauntlett 2011, p4). However, it falls short in explaining concurrent changes in physicality (Fitzgerald 2017). MBC tackles this issue by including physical capital alongside the economic, social, cultural and human subcategories which account for recovery capital. Taken from the work of Shilling (1991), physical capital refers to the social formation of bodies through sporting, leisure and other activities; it provides an ideal lens through which the potential benefits of exercise upon recovery can be articulated.

The MBC yoga programme was developed specifically for PF. During the sessions, the ability range of participants is used to pitch the level of intensity and posture difficulty; Sessions end by "entering savasana"; a meditative state of mindful relaxation. Mindfulness is a state of intentional non-judgmental attention regarding one's present-moment existence and experience (Kabat-Zinn et al. 2002). This is a particularly challenging, yet potentially positive (Appel and Kim-Appel 2009) mood state to achieve for those recently embroiled in the chaos of a substance use disorder (SUD). Exercises performed prior to entering savasana are designed to shut out external thoughts and centre service users in the 'now', making the transition to the meditative state smoother. Learning to use the body to calm down and selfregulate in this fashion is invaluable for those suffering the complex trauma often associated with SUD (Emerson et al. 2009). MBC rewards service users who attend six yoga sessions with a 6-month gym membership.

\section{Yoga and Addiction}

People in the throes of SUD often suffer social exclusion and reside at the fringes of society, where the disorder intersects with systemic inequality and long-term disadvantage. Individually, they are often found to suffer from anxiety, depression, low self-esteem and poor physical health (De Leon 2000). As such, they are a group for whom the potential benefits of exercise are considerable. Sustained and stable recovery stages occur at 1-5 and 5+ years respectively (The Betty Ford Institute Consensus Panel 2007). PF residents are considered to be in early recovery (0-1 year sober). Accordingly, a Rapid Evidence Assessment was conducted for 
empirical research published prior to December 2016, which explored the SUD/exercise relationship for active substance misusers or those in early-stage recovery.

Discounting studies focused on substance use rather than abuse, and animal research, 47 pieces of research were uncovered. Of these, 15 covered psychosocial outcomes; with all reporting positive findings of some sort. However, with the notable exceptions of Burling et al. (1992), Neale et al. (2012) and Landale and Roderick's (2014) emic (insider) focus, none shed light on the participant experience. Each study concluded that the explanatory power of substance and exercise type appeared to be superseded by levels of participant ownership and personal change. These findings are in alignment with PF and MBC values but it is important to note that they were absent from the 12 meta-analyses carried out investigating SUD and exercise (Read and Brown 2003; Greer et al. 2012; Weinstock et al. 2012; Williams and Strean 2004; Zschucke et al. 2012; Lynch et al. 2013; Wang et al. 2014; Giesen et al. 2015; Bardo and Compton 2015; Linke and Ussher 2015; Brellenthin and Koltyn 2016; Stoutenberg et al. 2015). This was due to an inclusion criterion favouring the positivist research paradigm, which discredits the SUD sufferers' voice as a source of credible data. Included findings range from significantly increased measurements of subjective wellbeing; self-perception, esteem (Palmer et al. 1988), internal locus of control (Ermalinski et al. 1997), self-cathexis (Gary and Guthrie 1972) and positive body image (Roessler 2010), to decreases in co-morbid conditions associated with an SUD; anxiety, depression (Palmer et al. 1995) and social phobia (Frankel and Murphy 1974; Palmer et al. 1988; Mamen et al. 2011; Rawson et al. 2015; Beitel et al. 2016; Unhjem et al. 2016).

Ermalinski et al. (1997) and Marefat et al. (2011) studies stand out from this evidence base as the only ones to use yoga. In these studies, participation was found to significantly reduce anxiety and depression (Marefat et al. 2011) and increase internal locus of control and responsibility for health when combined with incremental jogging (Ermalinski et al. 1997). Rehabilitative yoga within prisons has also been found to increase executive control while lowering levels of perceived stress (Bilderbeck et al. 2013); negatively impacting re-incarceration rates (Landau and Gross 2008) and decreasing depression and anxiety (Harner et al. 2010) among inmates for whom substance misuse is a recognized criminogenic pressure (Welch 2004).

\section{This Investigation}

This investigation focuses upon the MBC yoga programme in order to (1) Gauge the impact of therapeutic yoga classes upon the mood state of recovering participants and (2) Explore the perceived benefits of therapeutic yoga class participation. An adapted version of The Short Form Profile of Mood States (POMS-SF; Shacham 1983) described below, provided an effective measurement for before and after class mood. Ethnographic interviews were used to preserve the participant voice, gaining emic insight into the subjective experience of any service user who attended $6+$ classes.

\section{Methods}

\section{Design}

In carrying out this investigation it was important to remain consistent with the values of MBC and PF. The Community as Method approach, upon which PF is modelled, empowers service 
users to take ownership of their lives via emersion in a structured and supportive environment (Manning 1989; Kennard 2004). In this environment the skills of reflexivity; self-critique and self-appraisal are developed (Koch and Harrington 1998; Fitzgerald 2017). Human capital is routed in this reflexivity and command of the concepts and techniques often, but not always taught as part of rehabilitation that facilitates comprehension and articulation of the external world and one's position in it (Fitzgerald 2017).

The MBC therapeutic yoga programme complements this model by providing a safe space and an enjoyable experience through which participants are encouraged to exercise physical capital using their body to enter the meditative state of savasana. Therefore an intrusive, drawn-out gathering of data, before and after what can be a very spiritual experience for some, would likely impact negatively upon this.

Measures exist for the traditional recovery capital construct: The 50 item Assessment of Recovery Capital (Groshkova et al. 2013) has been successfully applied as a research tool, although it has been criticized for not accounting for community and having limited application within treatment settings and recovery organizations (Cano et al. 2017). The later 10-item Brief Assessment of Recovery Capital (Vilsaint et al. 2017) was created with busy workloads in treatment settings in mind, however, the same criticism regarding community applies. The REC-CAP (Best et al. 2016) represents an arguably more holistic assessment of potential barriers to recovery capital and includes a measurement for "meaningful activities" but this does not constitute physical capital and the tool itself is a 10-page battery of psychometric measurements. It is also the case that for participants in the fulltime residential PF programme, the skills which constitute human capital are being developed but many, particularly social and economic aspects of recovery capital, are likely to be held in stasis.

Rather than use these constructs, the "pragmatic" approach to research employed here entails converging different forms of data gained via mixed methods, selected to best suit the real word situation being studied (Creswell et al. 2003; Bergmann 2011). With a $1 \mathrm{hr}$ window for each class and the often low literacy of participants, the quick, easy and well-validated POMS (Curran et al. 1995) protocol with simple Likert scales was used to gather data in a stress-free and unobtrusive manner. Findings from the ethnographic interviews were converged with those from the statistical analysis of the POMS data. In a research area where the service users' voice is often absent, this approach is as warranted as it is innovative. It is also in keeping with the PF and MBC values of empowerment and community ownership.

\section{Sample}

Our sampling technique was purposive, focussing upon the two weekly non-compulsory yoga classes MBC runs for PF. One of these was for the "main" rehabilitation centre which takes place on-site and the other was for the "family" centre where residents stay with their children and occasionally spouses. These classes were held in a space provided by Sheffield Hallam University. At the beginning and end of each class, service users were asked if they would fill in the POMS questionnaire. Participation in data gathering was entirely voluntary. Our POMS response rate was $100 \%$, with no instances of attendees not wanting to complete the questionnaire. Data was gathered for 132 individual sessions from 34 participants (male; $N=17$, female; $N=17$ ). Thirty-three participants described themselves as "white British" and 1 described themselves as having "mixed heritage". While not entirely representative, this $97 \%$ white British sample reflects the fact that the vast majority $(85 \%)$ of clients in addiction treatment in England are white British with "other white" being the next most common (4\%) 
ethnicity (Public Health England 2017). No non-white ethnic group accounted for more than $1 \%$ of the total of addiction treatment during 2016-2017 (Public Health England 2017). The majority (68\%) of our sample was aged 30-34, with the youngest being 22 years and the oldest being 58 years. Eight participants completed at least six classes and went on to use their gym membership; from those eight, seven participants were interviewed (male; $N=2$, female; $N=$ 5). The eighth potential interviewee left treatment before an interview could be carried out.

\section{Measurements}

The POMS is a reliable and valid measurement tool that is widely used in clinical, psychological and sports research (Di Corrado 2017). Shortened versions of the POMS are available for research settings such as this one, in which, the full 65 item version is unsuitable (Curran et al. 1995). For this investigation, we adapted the 37-item Short Form (POMS-SF; Shacham 1983) to include Relaxation as one of seven mood subscales: Anger, Confusion, Depression, Fatigue, Tension, Vigour and Relaxation. Each subscale consisted of 4-6 single word items. A Cronbach's Alpha reliability analysis score of 0.82 indicated a very high level of inter-item correlation for our new Relaxation subscale single words. This is in line with the Cronbach Alpha scores for the other pre-existing six scales in Table 1 which range from $0.803-0.93$. We also substituted the Americanism "bushed" for "knackered" on the Fatigue subscale to better suit our British sample. Our Adapted Short Form (POMS-ASF) had 31 single word items in total. Respondents indicated how much they felt like each word immediately prior and immediately after each yoga session, using a Likert scale $(0=$ not at all; $1=$ a little; $2=$ moderately; 3 = quite a bit; 4 = extremely). Higher scores suggest a higher level of feeling. Following data collection, the single word items for each before and after subscale were computed into new variables to give an overall pre-yoga score and post-yoga score for each mood.

\section{Interviews}

Seven participants were ethnographically interviewed using an exploratory in-depth openended approach as advocated by Schensul et al. (1999). Gaining emic perspectives requires a good rapport and reflexive awareness on the part of the interviewer and interviewee (Westby 1990; Johnston et al. 1995). Therapeutic Community members are often ideal interviewees due to the reflective nature of the rehabilitation programme (Fitzgerald 2017). Our research team developed their understanding of the MBC philosophy and the participant experience by taking part in the range of $\mathrm{MBC}$ programmes. This intersubjective experience, interviewing

Table 1 Cronbach's alpha scores showing high levels of inter item correlation for each of the seven mood state subscales

\begin{tabular}{llll}
\hline Mood state & N of items & $N=$ & Cronbach's alpha \\
\hline Anger & 4 & 140 & .897 \\
Depression & 6 & 139 & .930 \\
Fatigue & 4 & 141 & .877 \\
Tension & 4 & 138 & .853 \\
Confusion & 4 & 140 & .856 \\
Vigour & 4 & 138 & .803 \\
Relaxation & 4 & 118 & .822 \\
\hline
\end{tabular}


technique and setting were designed to avoid laboratory conditions eliciting artificial responses to predetermined questions (Fetterman 1989; Johnston et al. 1995). As such, a certain level of rapport was ensured, which heightened the emic validity of our data. Interviews took place at the PF residential centres where residents live while "on program". Participants were free to introduce topics they felt were of importance to their SUD, rehabilitation and experience of yoga. Interviewers were free to word questions in whatever manner best fitted conversational flow and exercise reflexivity around sensitive and taboo subjects (Dickson-Swift et al. 2007).

\section{Analysis Technique}

Thematic analysis was employed in its "traditional form" as a method to draw themes from interview data (Braun and Clarke 2006). Successful ethnographies allow social action in one world to be understood by outsider cultures (Agar 1986); this understanding occurs via the emic perspective. Interview transcripts were repeatedly read and coded into themes by the lead interviewer and critically reviewed by the research team.

A comparison of means was carried out between pre and post-POM-ASF scores for each mood state with paired sample T-Test scores to determine statistical significance and the mean change over time, standardized into Cohen's D effect size statistics. Quantitative findings were split to highlight potential gender-based differences in outcome. Interview themes were triangulated with POMS-ASF findings, with equal value being given to quantitative and qualitative data. Abductive reasoning was applied to produce coherent findings (Onwuegbuzie and Leech 2006) of high internal validity. These findings were then discussed in relation to relevant literature with emphasis placed upon maintaining emic validity by preserving the voice of the participant as a source of credible knowledge.

\section{Results}

\section{POMS-ASF}

We found significant differences between the pre and post-yoga session scores for all but the Vigour mood state for both genders. The Cohen's D score for Fatigue denoted a small effect size for females and a small-medium effect size for males. The D values for Anger, Confusion, Depression and Tension indicated large differences in each mood state, whereas the Relaxation $\mathrm{D}$ value indicated a very large effect for both males and females (Tables 2 and 3).

\section{Thematic Analysis}

There were six connected universal themes to emerge from the interview data. The first two are the (1) Physical Improvements and (2) Psychological Improvements which the service users credit yoga with. Participants (3) Ascribed Therapeutic Value to Savasana, describing the calmness it entailed as a difficult state to achieve in day to day life. Participants described (4) Applying Yogic Techniques of Down Regulation Outside of the Sessions often to relax prior to sleep. Yoga is described as (5) Complementary to the PF Programme, particularly as an aid to self-reflection and a "timeout" from the weeks' therapeutic schedule. Related to this, all (6) Participants Planned to Continue Exercising Upon Completion of their PF Programme. 
Table 2 Comparison of means for males results

\begin{tabular}{llllllllll}
\hline Mood state & Pre mean & SD & Post mean & SD & Mean diff & N= & Pooled SD & Sig & Effect size \\
\hline Anger & 0.62 & 0.854 & 0.08 & 0.271 & -0.538 & 60 & 0.736 & $p<0.001$ & -0.73 \\
Depression & 0.91 & 1.139 & 0.14 & 0.523 & -0.768 & 57 & 1.070 & $p<0.001$ & -0.72 \\
Fatigue & 1.27 & 0.944 & 0.83 & 0.990 & -0.439 & 57 & 1.053 & $p<0.003$ & -0.42 \\
Tension & 0.70 & 0.882 & 0.11 & 0.309 & -0.589 & 56 & 0.692 & $p<0.001$ & -0.85 \\
Confusion & 0.82 & 0.888 & 0.16 & 0.336 & -0.665 & 56 & 0.807 & $p<0.001$ & -0.82 \\
Vigour & 1.56 & 0.672 & 1.68 & 0.626 & 0.118 & 57 & 0.880 & $\mathrm{p}<0.314$ & 0.13 \\
Relaxation & 1.57 & 0.634 & 2.95 & 0.863 & 1.385 & 50 & 0.684 & $p<0.001$ & 2.02 \\
\hline
\end{tabular}

Comparison of males pre and post means for each mood state with paired sample $T$ test scores indicating statistical significance for Anger, Depression, Fatigue, Tension, confusion, and relaxation but not for vigour. Cohen's D effect size statistics indicate; a small difference in Fatigue; a large difference in Anger, Confusion, Depression and Tension; and a very large difference in Relaxation

The Physical Improvements (1) discussed range from feeling better day to day to improved strength and/or mobility overtime.

David: "I'm able to stretch more, bend down lower, keep my body in the right position ... I've noticed a lot of um progression in that ... and [my] core's a lot stronger now". Charlotte: "When I first did it ... I couldn't even do a push up ... and now I know you have to put your hands to the side and like go down and then up".

Andrew: "My hips still do hurt a bit but not as much as they did when I first started, there's more flexibility and stuff ... I've noticed I can fold down more".

Jane: "Physically I was really, really, really restricted I was using a walking stick ... I basically thought [about yoga] no I can't do that but since Jo's [instructor] been coming I've taken part [and] I'm not taking any prescribed medication other than a couple of paracetamols maybe once or twice a week ... that's how being here [at PF] and having that yoga has transformed my life".

Jane placed great emphasis upon the reduction in pain she had experienced and the positive changes this had led to. Yoga has been associated with pain reduction in numerous studies (Büssing et al. 2012) but none with a recovery focus. Charlotte also experienced a reduction in pain, but this appeared to be related to relaxation.

Table 3 Comparison of means for females results

\begin{tabular}{llllllllll}
\hline Mood state & Pre mean & SD & Post mean & SD & Mean diff & N= & Pooled SD & Sig & Effect size \\
\hline Anger & 0.70 & 0.908 & 0.12 & 0.302 & -0.575 & 77 & 0.775 & $p<0.001$ & -0.74 \\
Depression & 1.30 & 1.582 & 0.50 & 1.049 & -0.805 & 77 & 0.862 & $p<0.001$ & -0.93 \\
Fatigue & 1.28 & 1.190 & 1.02 & 1.012 & -0.257 & 76 & 1.267 & $p<0.082$ & -0.20 \\
Tension & 0.83 & 0.740 & 0.24 & 0.495 & -0.594 & 77 & 0.626 & $p<0.001$ & -0.95 \\
Confusion & 0.88 & 0.882 & 0.32 & 0.582 & -0.561 & 78 & 0.699 & $p<0.001$ & -0.80 \\
Vigour & 1.31 & 0.862 & 1.25 & 1.043 & 0.053 & 75 & 0.810 & $p<0.609$ & 0.07 \\
Relaxation & 1.10 & 0.743 & 2.63 & 0.916 & 1.524 & 62 & 1.121 & $p<0.001$ & 1.36 \\
\hline
\end{tabular}

Comparison of females pre and post means for each mood state with paired sample $\mathrm{T}$ test scores indicating statistical significance for Anger, Depression, Fatigue, Tension, confusion and relaxation but not for vigour. Cohen's D effect size statistics indicate; a small difference in Fatigue; a large difference in Anger, Confusion, Depression and Tension; and a very large difference in Relaxation 
Charlotte: "It's like I go in with a massive headache that's ... a stress headache ... and then afterwards I just don't have one".

The other participants focused upon the Psychological Improvements (2) they ascribed to yoga rather than the physical improvements.

Andrew: "I feel really like refreshed. Relaxed in the mind I can feel a bit in the body but ... they make you feel ... at peace a bit more, just take away your worries a bit".

Katie: "I'm pushing myself and I feel good about it".

David: "I just get so much out of it you know, it just makes me feel really, really good, it makes me feel like healthy. I'm doing something positive it makes me feel positive like ... since I've been doing yoga... it's like every day I'm positive I'm grateful and I'm enthusiastic".

Sarah: "I've got a lot of stress, anxiety and stuff ... and I feel a lot more chilled and relaxed so it does have a positive real positive impact".

The potential overlap between Charlotte's relaxation and pain reduction alludes to an interconnectedness between physical and psychological benefits. There is the suggestion here that the psychological is being accessed through the physical and that down regulation is being achieved in the manner propagated by MBC.

Andrew: "When you get the peaceful mental side of it and then with the ... physical, they both come together ... and yeah you feel better a lot better ".

David: "If I've got any worries on my mind or anything, after the yoga session I'm just totally calm, relaxed, my mind feels good, my body feels good ... After these sessions I can feel ... a real progression in ... my ability ... which wasn't even my intention in the first place ... I feel really ... refreshed and quite alert funnily enough, even though my body feels ... like I've done something".

Jane: “After you've done that whole session you're just like wow that was amazing and physically you feel the difference but also mentally and emotionally you feel a big difference as well ... we do so much in that one hour ... after you've done it you feel really calm ... you feel really relaxed physically... it just puts your mind-set in a different place as well. It effects everything not just stretching your muscles but your attitude to things. You feel relaxed, you feel calm but equally you feel as if you've had a workout".

The interviewees did not explicitly state that the physical exertion of transitioning through yogic postures centred them in the moment, facilitating relaxation but, in Ascribing Therapeutic Value to Savasana (3), each discussed some form of mind body synergy suggesting an awareness of the relationship between the two.

David: "It's a really good way to end the yoga session because ... it brings your breathing down. You just slow everything down a little bit and you get into this kind of mental meditative state of ... calmness and peacefulness and you're able to reflect on the goodness you've done for your body and your mind ... amazing".

Andrew: "To get my heart [rate down] ... Jo's [instructor] brilliant at just calming it down and talking you through ... feeling stuff through your body ... from your toes all 
the way up and ... if you concentrate it's something you're able to do ... I'm getting better at that".

Sarah: "I think that's a nice feeling just to think about yourself for a while, think about what you're doing as you're going in and out of the moves and ... especially the relaxation bit as well ... Relaxing all your muscles ... I think that's what makes me be able to get up and feel energetic afterwards".

Charlotte: "I was laying there and I was thinking my body is so relaxed but my mind's still going but not ... so much".

The SUD/trauma association (Emerson et al. 2009) is of relevance here. Participants were not assessed for trauma-related disorders. It is the case, however, that approximately $46 \%$ of people with lifetime post-traumatic stress disorders have a comorbid SUD (Pietrzak et al. 2011) and that $80 \%$ of females entering treatment report traumatic pasts (Cohen et al. 2013). For Charlotte, down regulation was experienced as a slowing down of thoughts. David and Andrew described it in terms of bringing the heart rate down through breathing. Yoga has been empirically validated as efficacious in treating disorders involving stress reaction (Büssing et al. 2012) and Anderzén-Carlsson et al. (2014) found effectiveness to be based upon the ability to deal with stress outside of the yoga class. In Applying Yogic Techniques of Down Regulation Outside of the Sessions (4), our participants reported this.

Sarah: "Learning how to breath, relax and destress especially when we're here... it's needed cos we're in an environment where we're surrounded by each other so you don't get much alone time so it's difficult to find that time to destress so it's a simple technique that we've learned".

Mandy: "The deep breathing ... not just short, short breaths it's long deep long breaths from the stomach. It works for me when we're going to bed you know to just sort of concentrate on that and then I'll just drift off'.

Jane: "[I'm] more balanced in my life now because ... I'm able to deal with things mentally in a much more balanced and logical way".

The decision to enter treatment is often predicated by what is referred to in recovery circles as "hitting rock bottom" (Cunningham et al. 1994); a chaotic state in which individual action often intersects with systemic inequality and long-term disadvantage. Furthermore, hitting rock bottom is characterized by a breakdown of recovery capital. The Community as Method approach challenges habits and behaviours formed during this experience in order to overcome, manage or contain them (Phoenix Futures 2011). This application of down regulatory practices is an example of such change and, as such, is perhaps the most important finding of this research. It suggests that human capital, in the form of a reflexive awareness of arousal, is being exercised and that physical capital is exercised to down regulate.

The fifth theme was that all interviewees highlighted a Complimentary Fit between Yoga and the PF programme. Jane explicitly attributed her recovery to a combination of the two.

Jane: "To me it's something that should be here as part of the structure ... they've got the circuits [also an MBC program] ... they've got the RTN [Recovery Through Nature program] and that's really good for people that are further along in the programme but I 
think of welcome house [new PF residents], we need something inside of the project whereby people come in and do something with them and at the moment there's only the yoga ... that does that and if you took that away there's nothing".

For the family centre residents, yoga fitted well with the PF programme because it afforded them something that was missing from their programme; time away from child care responsibilities.

Sarah: "When I've got kids you know it's very difficult to plan ... so to have the opportunity to do yoga whilst I'm here and to get that time out, it's been important". Mandy: "It's about the only time of the week that [we] do get ... to think of ourselves because other times ... you need to think of the community you have to ...think of the kids".

For David, the techniques taught in yoga were conducive to the recovery process. He also explicitly mentions the transition from the physical exertion of postures to a meditative state of mind.

David: "We do meditation here and it kind of puts you in a similar frame of mind ... like the session of yoga we did last week ... we did repetitions of ... I can't remember the name of the move but we did loads of those and ... I got in this kind of meditation frame of mind and that's just brilliant cos it's just ... really peaceful ... It's really really good. Fits really well in with a recovery program ... [when] you've got a drug habit ... you're not really looking after your physical [self] or doing exercise or nothing like that ... so getting well should include some of that really".

The final theme covers Future Physical Exercise Plans (6). Each participant mentioned that they intended to either continue yoga, to begin other forms of exercise or a combination thereof.

David: "Yoga, it's giving me a sense of ... wellbeing and from that I've started to do other things cos I wanna get my body healthy cos it's making my mind feel healthy so as I said to you before I'm jogging now ... I haven't jogged for ... 25 years".

Katie: "I've been on about six sessions now and I've learned so I can even do it at home".

Andrew: "It's something that I wanna carry on with ... it makes you feel good, good about yourself ... you've got to remain healthy after what we've put ourselves through, and I wanna continue ... I'll give up smoking and get my lungs a bit better ... just keep fit ... just to be fit and healthy".

These statements constitute verbal commitments to a change in lifestyle which has the potential to be an important adjunct to SUD treatment. The challenge for those actively misusing substances and in early recovery is the implementation and initial sustaining of new exercise habits. The MBC yoga programme offers the opportunity to build physical capital during this period. 


\section{Discussion}

Our POMS-ASF findings suggest that regardless of gender, a single session of yoga significantly reduced Anger, Confusion, Depression, Fatigue, Tension, and significantly increased Relaxation in the present sample. Due to our predominantly white British sample we are unable to comment on the potential relationship between ethnicity and POMS-ASF findings for recovering addicts. It is, however, worth noting that preliminary data gathered by our research team for a study which replicates the methods outlined here in order to gauge the impact of therapeutic yoga classes upon the mood state of survivors of human trafficking from a range of ethnic backgrounds shows strikingly similar outcomes.

Through the application of abductive reasoning, interview data was converged with POMS-ASF findings, firstly to shed explanatory light upon them and secondly to highlight instances of construct validity, wherein outcomes related to yoga participation were inaccurately measured and/or entirely missed by the quantitative data gathering tool. For example, POMS-ASF findings tell us anxiety (tension) decreased whilst relaxation increased. Qualitative descriptions of entering sessions feeling stressed and leaving stress free expand upon this relationship between mood states. For our participants, it seems the panic, rooted in a lack of control which characterizes anxiety, must be reduced for relaxation to occur.

The experience of physical and psychological benefits, the therapeutic value of savasana, and the complimentary fit between therapeutic yoga and the PF programme were important to all interviewees. Directly testing the physical benefits related to yoga in future research would facilitate expansion regarding physical outcomes, however, such tests are expensive and potentially dangerous for cohorts, for whom, strenuous exercise is likely to be contraindicated (Noonan and Dean 2000). Submaximal testing can eliminate safety issues by producing sufficient exercise stress without facilitating biomechanical or physiological strain (Noonan and Dean 2000). Although this type of testing could negatively affect the inclusivity of a programme targeted at service users, for whom, the exercise environment is likely to be alien and frightening (Read et al. 2001; Abrantes et al. 2011). In the absence of such objective indicators, from the current investigation, we can still state with some confidence that yoga participation had positive physical outcomes for our participants. Self-perceived fitness does accurately correlate with objective measurements of fitness (Lamb 1992) and each of our interviewees perceived their fitness to have increased. It is also important to note that perceived fitness has been found to be a more accurate measurement of "daily coping" than actual fitness (Plante et al. 2000). There is potential for future research to build upon these findings firstly by exploring the interplay between perceived and actual fitness and also mood state for this cohort and secondly by measuring levels of flexibility and self-perceived pain. Before and after "sit and reach" measurements represent a safe and accurate way to quantify changes in flexibility (Baltaci et al. 2003) and perceived pain can be measured via quantitative sensory testing (Yarnitsky and Fowler 1997). We recommend that data gathering for such research should however, remain as unobtrusive as possible, firstly because exercise is likely to be challenging for those early on in their recovery and secondly because insensitive data collecting would likely spoil the therapeutic setting in an MBC session.

The interview data expands upon the significant decreases in Anger, Depression, Tension and Confusion reported via POMS. Anti-depressive and anxiolytic outcomes are generally associated with yoga (Da Silva et al. 2009) and have been observed in the 
recovery community (Marefat et al. 2011). In this instance, these outcomes appear mediated by classes representing time out from intense rehabilitation programmes. This was particularly the case for family service residents who have the added responsibility of child care. Savasana plays an important role here, with participant descriptions of a meditative state of calmness and peacefulness in which the mind has slowed down. This suggests breathing and flowing between postures is indeed shutting out external thoughts, allowing a smoother transition into a state of relaxation. Interviewees attributed increased energy levels following classes directly to this process, despite POMS-ASF results suggesting no significant difference between pre and post-intervention vigour scores. This suggests that the POMS-ASF may not be accurately measuring vigour. We postulate this may be related to the introduction of a Relaxed subcategory which included words such as "chilled". Participants counter-intuitively scoring themselves as highly "chilled" yet simultaneously highly "energetic" may be in effect.

Participants suggested that yoga is a form of exercise which is readily accessible to new, often detoxing residents at PF's main rehab centre due to it being on-site and less physically demanding than other activities. Focusing specifically upon the early recovery period of detoxification and the potential impact of the $\mathrm{MBC}$ programme is one direction our future research shall take.

What the interviews revealed, that the POMS-ASF did not, is firstly that savasana seems to be easier to reach the more sessions a participant has completed and secondly that once learnt, such down regulation can be achieved outside of yoga. Phrased using the revised recovery capital framework, our participants described exercising human capital and physical capital; using the physical to regulate the psychological. To centre oneself in this manner is to exercise recovery capital, a finding which supports Landau and Gross (2008) and Anderzén-Carlsson et al. (2014), highlighting the transferability of the practices of yoga. This is important as it suggests that the efficacy of the MBC yoga programme, as an adjunctive treatment to the PF rehabilitation model, lies partially in the learning of transferable skills with the potential to aid recovery.

Our interviewees' desire to continue exercising suggests that doing so has the potential to become an aspect of their recovery capital on a mid to long-term basis. The fact that $80 \%$ of those in sustained (1-5 years) and $77 \%$ of those in stable (5+ years) recovery, exercise regularly (Best et al. 2013) suggests that doing so correlates with prolonged recovery. For those whom exercise has never been a meaningful activity (such as Jane), regularity would represent new-found physical capital. For others (such as David and Andrew) the embedding of exercise as part of weekly routine would represent using physical capital to aid the "reaching back to a pre-addiction state" which lies at the heart of recovery (Irving 2011). MBC often remains in contact with PF residents after they complete their rehabilitation programme and we plan to investigate the mid to long-term exercise habits of $\mathrm{MBC}$ service users in the near future.

\section{Limitations}

This study has the following limitations: Firstly, there was no control group. It is conceivable although unlikely that PF residents who chose not to participate in the yoga classes also had significant decreases in negative and increases in positive mood states during equivalent $1 \mathrm{~h}$ periods. For this reason, we cannot categorically assign mood state changes to yoga participation but, given that the anti-depressant and anxiolytic value of yoga for populations for whom 
substance misuse is a recognized criminogenic pressure has been confirmed (Harner et al. 2010) and that our POMS-ASF findings have been expanded upon by interviewees, we can strongly infer that yoga was the driver of positive mood state change for our participants. Secondly, it would have been prudent to interview residents who completed less than six classes to assess their thoughts for comparison with participants who had attended six or more sessions and POMS-ASF findings. Setting up exercise-based interventions in therapeutic communities can be challenging because above all the community must continue to function and individual rehabilitation programmes with associated resident responsibilities must be adhered to (Fitzgerald 2017). The potential reasons behind a resident attending less than six yoga classes range from personal taste to disciplinary sanction and extra hands being required to carry out household tasks. It is the case however, that without data pertaining to individual reasons for participant attrition, our emic accounts restricted to those who became invested in the classes.

\section{Conclusion}

The MBC yoga programme significantly reduced Anger, Confusion, Depression, Fatigue, and Tension, while significantly increasing Relaxation in our sample, without gender being a factor. Interview data suggests an increase in Vigour, despite inconclusive POMS-ASF findings. These psychological outcomes appear intertwined with the perceived physical benefits and relate specifically to savasana. Testing fitness submaximally alongside flexibility and self-perceived pain has been highlighted as a potential approach to operationalizing changes in physicality for future research, although we urge caution and recommend that all future projects be carried out in a manner conducive with PF's therapeutic and inclusive environment. Our data suggests that the $\mathrm{MBC}$ yoga programme can be a useful adjunctive to the PF programme given that interviewees described the down regulatory practices learned in yoga as being beneficial to their recovery. The revised recovery capital model provided a useful set of conceptual tools to discuss this and would also provide an ideal lens through which to explore the mid to long-term PE habits of the recovery community in general and $\mathrm{MBC}$ service users in particular. Another interesting direction for future research would be to focus specifically upon the early recovery period of detoxification and the potential impact of the yoga using the same theoretical model.

\section{Compliance with ethical standards}

This investigation met MBC's rigorous ethical standards. Neither SUDs nor associated taboo behaviours were the focus of the investigation underpinning this paper. As such concerns regarding possible psychological trauma related to the interview topic were greatly reduced. The physical saftey of participants during yoga classes was the resopnsibility of MBC's qualified and insured practitioners. In order to honour particpant confidentiality, aliases have been used and other identifiying details altered or removed.

Declaration of Interest Christopher Fitzgerald, Ruth Barley and Joanna Hunt are founding members of Mind Body Connect (MBC). Christopher Fitzgerald and Ruth Barley are Lecturers at the Department of Psychology, Sociology and Politics at Sheffield Hallam University. Joanna Hunt is an undergraduate student at Sheffield Hallam University studying Occupational Therapy. At the time this research was carried out she was in her first year of study. During the same period, Sini-Petriina Klasto and Rosie West were post-graduate students at The University of Sheffield. Following the completion of this research, Sini-Petriina Klasto and Rosie West became trustees for MBC. They are now completing doctorates at the University of Sheffield and Keele University respectively. 
Open Access This article is licensed under a Creative Commons Attribution 4.0 International License, which permits use, sharing, adaptation, distribution and reproduction in any medium or format, as long as you give appropriate credit to the original author(s) and the source, provide a link to the Creative Commons licence, and indicate if changes were made. The images or other third party material in this article are included in the article's Creative Commons licence, unless indicated otherwise in a credit line to the material. If material is not included in the article's Creative Commons licence and your intended use is not permitted by statutory regulation or exceeds the permitted use, you will need to obtain permission directly from the copyright holder. To view a copy of this licence, visit http://creativecommons.org/licenses/by/4.0/.

\section{References}

Abrantes, A. M., Battle, C. L., Strong, D. R., Ing, E., Dubreuil, M. E., Gordon, A., \& Brown, R. A. (2011). Exercise preferences of patients in substance abuse treatment. Mental Health and Physical Activity, 4(2), 79-87.

Agar, M. H. (1986). Speaking of ethnography (Vol. 2). Sage.

Anderzén-Carlsson, A., Persson Lundholm, U., Köhn, M., \& Westerdahl, E. (2014). Medical yoga: another way of being in the world - a phenomenological study from the perspective of persons suffering from stressrelated symptoms. International Journal of Qualitative Studies on Health and Well-Being, 9(1), 23033.

Appel, J., \& Kim-Appel, D. (2009). Mindfulness: implications for substance abuse and addiction. International Journal of Mental Health and Addiction, 7(4), 506.

Baltaci, G., Un, N., Tunay, V., Besler, A., \& Gerçeker, S. (2003). Comparison of three different sit and reach tests for measurement of hamstring flexibility in female university students. British Journal of Sports Medicine, 37(1), 59-61.

Bardo, M. T., \& Compton, W. M. (2015). Does physical activity protect against drug abuse vulnerability? Drug and Alcohol Dependence, 153, 3-13.

Beitel, M., Stults-Kolehmainen, M., Cutter, C. J., Schottenfeld, R. S., Eggert, K., Madden, L. M., et al. (2016). Physical activity, psychiatric distress, and interest in exercise group participation among individuals seeking methadone maintenance treatment with and without chronic pain. The American Journal on Addictions, 25(2), 125-131.

Bergmann, M. M. (2011). The politics, fashions, and conventions of research methods. Journal of Mixed Methods Research, 5, 99-102.

Best, D., \& Laudet, A. (2010). The potential of recovery capital. London: RSA.

Best, D., Savic, M., Beckwith, M., Honor, S., Karpusheff, J., \& Lubman, D. I. (2013). The role of abstinence and activity in the quality of life of drug users engaged in treatment. Journal of Substance Abuse Treatment, 45(3), 273-279.

Best, D., Edwards, M., Mama-Rudd, A., Cano, I., \& Lehman, J. (2016). Measuring an individual's recovery barriers and strengths. Addiction professional.

Betty Ford Institute Consensus Panel. (2007). What is recovery? A working definition from the Betty Ford Institute. Journal of Substance Abuse Treatment, 33(3), 221.

Bilderbeck, A. C., Farias, M., Brazil, I. A., Jakobowitz, S., \& Wikholm, C. (2013). Participation in a 10-week course of yoga improves behavioural control and decreases psychological distress in a prison population. Journal of Psychiatric Research, 47(10), 1438-1445.

Braun, V., \& Clarke, V. (2006). Using thematic analysis in psychology. Qualitative Research in Psychology, 3(2), 77-101.

Brellenthin, A. G., \& Koltyn, K. F. (2016). Exercise as an adjunctive treatment for cannabis use disorder. The American Journal of Drug and Alcohol Abuse, 42(5), 481-489.

Burling, T. A., Seidner, A. L., Robbins-Sisco, D., Krinsky, A., \& Hanser, S. B. (1992). Batter up! Relapse prevention for homeless veteran substance abusers via softball team participation. Journal of Substance Abuse, 4(4), 407-413.

Büssing, A., Ostermann, T., Lüdtke, R., \& Michalsen, A. (2012). Effects of yoga interventions on pain and painassociated disability: a meta-analysis. The Journal of Pain, 13(1), 1-9.

Cano, I., Best, D., Edwards, M., \& Lehman, J. (2017). Recovery capital pathways: modelling the components of recovery wellbeing. Drug and Alcohol Dependence, 181, 11-19.

Cloud, W., \& Granfield, R. (2008). Conceptualizing recovery capital: expansion of a theoretical construct. Substance Use \& Misuse, 43(12-13), 1971-1986.

Cohen, L. R., Field, C., Campbell, A. N., \& Hien, D. A. (2013). Intimate partner violence outcomes in women with PTSD and substance use: a secondary analysis of NIDA clinical trials network "women and trauma" multi-site study. Addictive Behaviours, 38(7), 2325-2332.

Creswell, J. W., Plano Clark, V. L., Guttmann, M. L., \& Hanson, E. E. (2003). Advanced mixed methods research design. In A. Tashakkori \& C. Teddlie (Eds.), Handbook of mixed methods in social and behavioral research (pp. 209-240). Thousand Oaks: Sage. 
Cunningham, J. A., Sobell, L. C., Sobell, M. B., \& Gaskin, J. (1994). Alcohol and drug abusers' reasons for seeking treatment. Addictive Behaviours, 19(6), 691-696.

Curran, S. L., Andrykowski, M. A., \& Studts, J. L. (1995). Short form of the profile of mood states (POMS-SF): psychometric information. Psychological Assessment, 7(1), 80.

Da Silva, T. L., Ravindran, L. N., \& Ravindran, A. V. (2009). Yoga in the treatment of mood and anxiety disorders: a review. Asian Journal of Psychiatry, 2(1), 6-16.

De Leon, G. (2000). The therapeutic community: theory, model, and method. New York: Springer Publishing Co.

Di Corrado, D. (2017). Biological underpinnings of mood and the role of physical exercise. Sport Sciences for Health, 13(3), 461-468.

Dickson-Swift, V., James, E. L., Kippen, S., \& Liamputtong, P. (2007). Doing sensitive research: what challenges do qualitative researchers face? Qualitative Research, 7(3), 327-353.

Emerson, D., Sharma, R., Chaudhry, S., \& Turner, J. (2009). Trauma-sensitive yoga: principles, practice, and research. International Journal of Yoga Therapy, 19(1), 123-128.

Ermalinski, R., Hanson, P. G., Lubin, B., Thornby, J. I., \& Nahormek, P. A. (1997). Impact of a body-mind treatment component on alcoholic inpatients. Journal of Psychosocial Nursing and Mental Health Services, 35(7), $39-45$.

Fetterman, D. M. (1989). Applied social research methods series: Vol. 17. Ethnography: Step by step.

Fitzgerald, C. (2017). Capitalising upon the physical: exercise and addiction recovery. (Unpublished doctoral thesis). University of Sheffield, Sheffield, United Kingdom.

Frankel, A., \& Murphy, J. (1974). Physical fitness and personality in alcoholism: canonical analysis of measures before and after treatment. Quarterly Journal of Studies on Alcohol, 35(4), 1272-1278.

Gary, V., \& Guthrie, D. (1972). The effect of jogging on physical fitness and self-concept in hospitalized alcoholics. Quarterly Journal of Studies on Alcohol, 33(4-A), 1073-1078.

Gauntlett, D. (2011). Three approaches to social capital. Supplementary text to Making is connecting: the social meaning of creativity, form DIY and knitting to YouTube and Web 2.0.

Giesen, E. S., Deimel, H., \& Bloch, W. (2015). Clinical exercise interventions in alcohol use disorders: a systematic review. Journal of Substance Abuse Treatment, 52, 1-9.

Greer, T. L., Ring, K. M., Warden, D., Grannemann, B. D., Church, T. S., Somoza, E., Blair, S. N., Szapocznik, J., Stoutenberg, M., Rethorst, C., \& Walker, R. (2012). Rationale for using exercise in the treatment of stimulant use disorders. The Journal of Global Drug Policy and Practice, 6(1).

Groshkova, T., Best, D., \& White, W. (2013). The assessment of recovery capital: properties and psychometrics of a measure of addiction recovery strengths. Drug and Alcohol Review, 32, 187-194.

Harner, H., Hanlon, A. L., \& Garfinkel, M. (2010). Effect of Iyengar yoga on mental health of incarcerated women: a feasibility study. Nursing Research, 59(6), 389-399.

Irving, A. D. (2011). Life story narratives of recovery from dependent drug and alcohol use: a tool for identity reconstruction within a therapeutic community. Therapeutic Communities: the International Journal of Therapeutic Communities, 32(3), 182-200.

Johnston, R. J., Weaver, T. F., Smith, L. A., \& Swallow, S. K. (1995). Contingent valuation focus groups: insights from ethnographic interview techniques. Agricultural and Resource Economics Review, 24(1), 56-69.

Kabat-Zinn, J., Segal, Z. V., Williams, J. M. G., \& Teasdale, J. D. (2002). Mindfulness-based cognitive therapy: a new approach to preventing relapse. New York: Guilford.

Kennard, D. (2004). The therapeutic community as an adaptable treatment modality across different settings. Psychiatric Quarterly, 75(3), 295-307.

Koch, T., \& Harrington, A. (1998). Reconceptualising rigour: the case for reflexivity. Journal of Advanced Nursing, 4(4), 882-890.

Lamb, K. L. (1992). Correlates of self-perceived fitness. Perceptual and Motor Skills, 74(3), 907-914.

Landale, S., \& Roderick, M. (2014). Recovery from addiction and the potential role of sport: using a life-course theory to study change. International Review for the Sociology of Sport, 49(3-4), 468-484.

Landau, P. S., \& Gross, J. B. (2008). Low reincarceration rate associated with Ananda Marga yoga and meditation. International Journal of Yoga Therapy, 18(1), 43-48.

Linke, S. E., \& Ussher, M. (2015). Exercise-based treatments for substance use disorders: evidence, theory, and practicality. The American Journal of Drug and Alcohol Abuse, 41(1), 7-15.

Lynch, W. J., Peterson, A. B., Sanchez, V., Abel, J., \& Smith, M. A. (2013). Exercise as a novel treatment for drug addiction: a neurobiological and stage-dependent hypothesis. Neuroscience \& Biobehavioral Reviews, 37(8), 1622-1644.

Mamen, A., Pallesen, S., \& Martinsen, E. W. (2011). Changes in mental distress following individualized physical training in patients suffering from chemical dependence. European Journal of Sport Science, 11(4), $269-276$.

Manning, N. (1989). The therapeutic community movement: charisma and routinisation. London: Routledge.

Marefat, M., Peymanzad, H., \& Alikhajeh, Y. (2011). The study of the effects of yoga exercises on addicts' depression and anxiety in rehabilitation period. Procedia-Social and Behavioral Sciences, 30, 1494-1498. 
Neale, J., Nettleton, S., \& Pickering, L. (2012). Heroin users' views and experiences of physical activity, sport and exercise. International Journal of Drug Policy, 23(2), 120-127.

Noonan, V., \& Dean, E. (2000). Submaximal exercise testing: clinical application and interpretation. Physical Therapy, 80(8), 782-807.

Onwuegbuzie, A. J., \& Leech, N. L. (2006). Linking research questions to mixed methods data analysis procedures 1. The Qualitative Report, 11(3), $474-498$.

Palmer, J., Vacc, N., \& Epstein, J. (1988). Adult inpatient alcoholics: physical exercise as a treatment intervention. Journal of Studies on Alcohol, 49(5), 418-421.

Palmer, J. A., Palmer, L. K., Michiels, K., \& Thigpen, B. (1995). Effects of type of exercise on depression in recovering substance abusers. Perceptual and Motor Skills, 80(2), 523-530.

Phoenix Futures. (2011). The manual: staff and community members' manual.

Pietrzak, R. H., Goldstein, R. B., Southwick, S. M., \& Grant, B. F. (2011). Prevalence and axis I comorbidity of full and partial posttraumatic stress disorder in the United States: results from wave 2 of the National Epidemiologic Survey on alcohol and related conditions. Journal of Anxiety Disorders, 25, 456-465.

Plante, T. G., Le Captain, S. E., \& McLain, H. C. (2000). Perceived fitness predicts daily coping better than physical activity. Journal of Applied Biobehavioral Research, 5(1), 66-79.

Public Health England. (2017). Adult substance misuse statistics from the National Drug Treatment Monitoring System. London: Public Health England.

Rawson, R. A., Chudzynski, J., Gonzales, R., Mooney, L., Dickerson, D., Ang, A., et al. (2015). The impact of exercise on depression and anxiety symptoms among abstinent methamphetamine-dependent individuals in a residential treatment setting. Journal of Substance Abuse Treatment, 57, 36-40.

Read, J.P., Brown, R.A., Marcus, B.H., Kahler, C.W., RAmsey, S.E., Dubreuil, M.E., Jakicic, J.M and Francione, C., (2001). Exercise attitudes and behaviours amoung persons in treatment for alcohol use disorders. Journal of Substance Abuse Treatment, 21(4), p.49.

Read, J. P., \& Brown, R. A. (2003). The role of physical exercise in alcoholism treatment and recovery. Professional Psychology: Research and Practice, 34(1), 49.

Roessler, K. K. (2010). Exercise treatment for drug abuse-a Danish pilot study. Scandinavian Journal of Public Health, 38(6), 664-669.

Schensul, S. L., Schensul, J. J., \& Le Compte, M. D. (1999). Essential ethnographic methods: Observations, Interviews, and Questionnaires (Vol. 2). Rowman Altamira.

Shacham, S. (1983). A shortened version of the profile of mood states. Journal of Personality Assessment, 47(3), 305-306.

Shilling, C. (1991). Educating the body: physical capital and the production of social inequalities. Sociology, 25(4), 653-672.

Stoutenberg, M., Warne, J., Vidot, D., Jimenez, E., \& Read, J. P. (2015). Attitudes and preferences towards exercise training in individuals with alcohol use disorders in a residential treatment setting. Journal of Substance Abuse Treatment, 49, 43-49.

Unhjem, R., Flemmen, G., Hoff, J., \& Wang, E. (2016). Maximal strength training as physical rehabilitation for patients with substance use disorder; a randomized controlled trial. BMC Sports Science, Medicine and Rehabilitation, 8(1), 7.

Vilsaint, C. L., Kelly, J. F., Bergman, B. G., Groshkova, T., Best, D., \& White, W. (2017). Development and validation of a brief assessment of recovery capital (BARC-10) for alcohol and drug use disorder. Drug and Alcohol Dependence, 177, 71-76.

Wang, D., Wang, Y., Wang, Y., Li, R., \& Zhou, C. (2014). Impact of physical exercise on substance use disorders: a meta-analysis. PLoS One, 9(10), 110728.

Weinstock, J., Wadeson, H. K., \& VanHeest, J. L. (2012). Exercise as an adjunct treatment for opiate agonist treatment: review of the current research and implementation strategies. Substance Abuse, 33(4), 350-360.

Welch, M. (2004). Ironies of imprisonment. London: Sage.

Westby, C. E. (1990). Ethnographic interviewing: asking the right questions to the right people in the right ways. Journal of Childhood Communication Disorders, 13(1), 101-111.

Williams, D. J., \& Strean, W. B. (2004). Physical activity as a helpful adjunct to substance abuse treatment. Journal of Social Work Practice in the Addictions, 4(3), 83-100.

Yarnitsky, D., \& Fowler, C. J. (1997). Quantitative sensory testing. Muscle \& Nerve, 20(2), 198-204.

Zschucke, E., Heinz, A., \& Ströhle, A. (2012). Exercise and physical activity in the therapy of substance use disorders. The Scientific World Journal, 2012, 1-19.

Publisher's Note Springer Nature remains neutral with regard to jurisdictional claims in published maps and institutional affiliations. 